\title{
Pronounced dependence of ventricular endocardial QRS potentials on ventricular volume ${ }^{1}$
}

\author{
JON LEKVEN, ${ }^{2}$ KANU CHATTERJEE, JOHN V. TYBERG, ${ }^{3}$ DAVID F. STOWE, ${ }^{4}$ \\ DETLEF G. MATHEY,5 AND WILLIAM W. PARMLEY
}

From the Department of Medicine, Cardiovascular Division and the Cardiovascular Research Institute of the University of California, San Francisco, Ca, USA

SUMMARY To evaluate the relation between ventricular endocardial potentials (QRS amplitude) and ventricular dimensions, left and right ventricular endocardial potentials were recorded with hook electrodes in anaesthetised open-chest dogs during transfusion and withdrawal of blood. Left ventricular end-diastolic diameter was measured by ultrasonic crystals, and end-diastolic volume was determined by thermodilution. In each dog, left ventricular endocardial potentials, whether recorded from anterior or posterolateral walls, decreased linearly as left ventricular end-diastolic diameter or volume increased, and vice versa. With an average increase in left ventricular end-diastolic diameter from $40.1 \pm 1.7$ to $44.6 \pm 1.8 \mathrm{~mm}$, left ventricular endocardial potentials decreased from $32.8 \pm 2.5$ to $23.5 \pm 2.3$ $\mathrm{mV}(\mathrm{P}<0.001)$; and for an increase in left ventricular end-diastolic volume from $1.36 \pm 1.25$ to $3.43 \pm$ $0.58 \mathrm{ml} / \mathrm{kg}$ left ventricular endocardial potentials decreased from $36.2 \pm 6.6$ to $14.9 \pm 4.3 \mathrm{mV}$ (P< 0.001). Changes in right ventricular endocardial potentials paralleled the changes in left ventricular endocardial potentials. These findings indicate that acute changes in ventricular dimensions influence endocardial potentials considerably, and suggest a potential clinical application for detecting acute changes in ventricular volume.

It has been previously reported that right ventricular endocardial potentials (i.e. the absolute magnitude of the endocardial QRS amplitude) decrease in the acute phase of myocardial infarction (Chatterjee et al., 1968; Parker et al., 1969; Chatterjee and Rouse, 1971). Furthermore, the more severe the clinical failure complicating acute myocardial infarction, the greater the decrease (Chatterjee et al., 1970). In experimental myocardial infarction a

\footnotetext{
${ }^{1}$ This work was supported in part by a NHLBI Program Project Grant.

${ }^{2}$ Recipient of a USPHS Fogarty International Fellowship. Present address: Institute for Experimental Medical Research, Oslo 1, Norway.

${ }^{3}$ Recipient of a USPHS Research Career Development Award and Grants-in-Aid from the American Heart Association and the Bay Area Heart Research Committee.

'Present address: Department of Physiology, Medical College of Wisconsin, 561 No. 15th St., Milwaukee, Wisconsin 53233, U.S.A.

'Present address: Department of Cardioangiology, University of Eppendorf, Martini Str. 52, 2000 Hamburg 20, West Germany.
}

Received for publication 24 October 1977. decrease in both right ventricular endocardial potential and left ventricular endocardial potential has been observed (Chatterjee and Rouse, 1971). This occurred in exploring electrodes placed both in the infarcted myocardium and at sites remote from the area of infarction. There was also some correlation between the magnitude of decrease in endocardial potential and the severity of depression of left ventricular function (Chatterjee and Rouse, 1971).

A decrease in right ventricular endocardial potential has also been observed in patients with acute massive pulmonary embolism complicated by severe right heart failure (Chatterjee et al., 1972). In both myocardial infarction and massive pulmonary embolism there are probably acute changes in ventricular volumes. The purpose of this study, therefore, was to investigate the direct influence of acute changes in ventricular volumes and dimensions on the magnitude of right and left ventricular endocardial potentials. Results indicate that there is an inverse relation between changes in endocardial potential and in ventricular volumes. 


\section{Materials and methods}

Changes in ventricular endocardial potentials were recorded in 18 open-chest mongrel dogs weighing 17 to $22 \mathrm{~kg}$. Anaesthesia was induced with intravenous sodium thiopentone $(25 \mathrm{mg} / \mathrm{kg})$. The dogs were then given intravenous morphine sulphate (45 mg) followed by maintenance doses of $15 \mathrm{mg} / \mathrm{hr}$. A continuous infusion of succinyl choline chloride, $20 \mathrm{mg} / \mathrm{kg}$ per $\mathrm{hr}$ was used for muscle relaxation. Respiration was maintained with a positive pressure respirator (Harvard Apparatus, Millis, Mass.) and regulated according to changes in arterial $\mathrm{Po}_{2}, \mathrm{PCO}_{2}$, and $p \mathrm{H}$, determined frequently (BMS $3, M K 2$, Radiometer, Copenhagen, Denmark) throughout the experiments. The heart was exposed by thoracotomy in the left fifth intercostal space. The anterior pericardium was opened and the margins secured to the lateral chest wall to form a pericardial cradle.

Right and left ventricular pressures were measured with solid state pressure transducers (Konigsberg Instruments, Pasadena, Calif.) introduced through the respective atrial appendages. Absolute values of diastolic pressure were ascertained by comparison with the tracing registered from the ventricles by a Statham P23 Db transducer connected to a 23 gauge needle by a stiff cannula. Aortic pressure was measured by a femoral catheter and an external transducer (P23 Db, Statham Instruments, Inc.). Aortic flow was measured with an electromagnetic flowmeter (Carolina Medical Electronics, King, North Carolina) on the ascending aorta.

Unipolar electrograms were recorded with the use of hook electrodes $(0.13 \mathrm{~mm}$ diameter, Elgiloy, Elgiloy Co., Elgin, Ill.), using the Wilson central terminal of extremity leads as a reference. Electrodes were insulated except for the hook portion which was $3 \mathrm{~mm}$ long. Electrodes were first introduced into the ventricular cavity through a 23 gauge needle. When the needle was removed the electrodes were attached to the endocardial surface and ST segment elevation due to endocardial contact was observed in the electrogram. Left ventricular endocardial potential was recorded from several endocardial sites on the left ventricular anterolateral wall, and in 3 dogs from the posterolateral wall also. Band pass filters were adjusted to exclude frequencies below $0.5 \mathrm{~Hz}$ and above $100 \mathrm{~Hz}$. Endocardial potential was read as the average in 4 consecutive beats. The sum of the highest positive and lowest negative components of QRS was regarded as the endocardial potential. Ectopic beats or QRS complexes showing conduction disturbances were excluded. The positions of the endocardial electrodes were verified at necropsy by dissecting the hearts.
In 7 dogs left ventricular volumes were calculated by thermodilution technique. A rapidly responding thermistor (time constant $50 \mathrm{~ms}$ ) mounted on the end of a curved spring (Wilton Webster, Inc., Altadena, Calif.) was introduced through a femoral artery to a position just above the aortic valve. Rapid injection of 3 to $4 \mathrm{ml}$ iced saline was made into the left ventricle via a catheter passed through the left atrial appendage. The thermistor position was manipulated until injection of iced saline produced discrete steps in the temperature record. Ratios of these successive steps were averaged and using the value of the stroke volume determined by the flowmeter, the value of left ventricular enddiastolic volume (LVEDV) was determined from the equation:

$$
\mathrm{EDV}=\mathrm{SV} /\left(1-\frac{\Delta \mathrm{T}_{\mathrm{n}}+1}{\Delta \mathrm{T}_{\mathrm{n}}}\right)
$$

where $\Delta T_{n}+1 / \Delta T_{n}$ is the ratio of the temperature deflections recorded in the aorta during successive beats and SV is stroke volume (Holt, 1956).

In 11 dogs anteroposterior left ventricular diameter was determined with an ultrasonic dimension recording system (Theroux et al., 1974). Piezoelectric crystals (diameter 4 to $5 \mathrm{~mm}$ ) were inserted into the myocardium through stab wounds in the anterior and posterior walls of the left ventricle. Thus, a minor axis of the left ventricle was continuously recorded.

After control measurements were performed, ventricular volume or diameter was increased by stepwise transfusion of donor blood from a reservoir through a wide bore cannula inserted into an external jugular vein. After each volume increment endocardial potentials and haemodynamic measurements were recorded. After achieving an enddiastolic pressure of 15 to $25 \mathrm{mmHg}$, the circulation was unloaded decrementally, repeating the measurements at each level. In 5 dogs the heart rate was kept constant by right atrial pacing during the experiment. In $1 \mathrm{dog}$, an exchange transfusion of saline against blood was done in order to reduce the dog's haematocrit, keeping the left ventricular diameter constant.

The data at control conditions and during blood transfusion and withdrawal were digitised and processed for computer analysis, using the Statistical Package for the Social Sciences (Nie et al., 1975). The subprogrammes, 'breakdown', 't-test', 'scattergram', and 'regression' were used in the Tables and Fig. 4, 5, 6, and 8. A P value greater than 0.05 was regarded as not statistically significant. To normalise for the expected variations in absolute values of endocardial potentials, left ventricular volumes and dimensions in different animals, the data were 
LV
endocardial
potential

LV
pressure

RV
pressure

LV

pressure

LV diameter
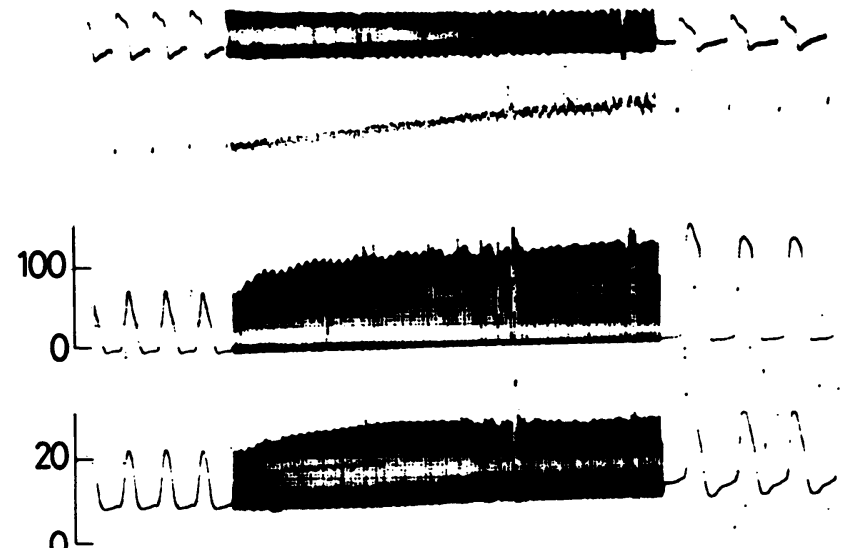

Fig. 1 Decrease in left ventricular endocardial potential associated with increase in left ventricular ( $L V)$ end-diastolic diameter during transfusion.

Increments in left and right ventricular pressures during transfusion are also shown. treated statistically by the Fisher z-transformation (Zar, 1974). The z-transformation involves subtracting the mean value of the parameter from the individual value and dividing the difference by the standard deviation, thus normalising betweenelectrode variance of endocardial potentials and between-dog variance of ventricular volumes and diameters.

\section{Results}

LEFT VENTRICULAR DIASTOLIC DIAMETER AND ENDOCARDIAL POTENTIALS

In 11 dogs, changes in left ventricular endocardial potentials and left ventricular end-diastolic diameters were recorded during transfusion and blood withdrawal. As left ventricular end-diastolic dimensions increased during transfusion, left ventricular endocardial potentials decreased (Fig. 1). Fig. 2 shows changes in the magnitude of left ventricular endocardial potentials, recorded from three different sites in one representative experiment, in relation to changes in left ventricular end-diastolic dimensions induced by blood transfusion and withdrawal. The absolute magnitudes of left ventricular endocardial potentials recorded from different sites were variable; however, left ventri- cular endocardial potentials recorded from each site decreased linearly as left ventricular end-diastolic dimension increased. The absolute magnitude of left ventricular endocardial potentials also varied in different dogs. Changes in the potentials recorded from 41 sites in 11 dogs relative to percentage change in left ventricular end-diastolic dimension during transfusion and withdrawal of blood are shown in Fig. 3. Though there was a considerable variation in absolute magnitudes of left ventricular endocardial potentials in different dogs, and when recorded from different sites (range 14 to $49 \mathrm{mV}$, average $32.8 \mathrm{mV}$ ), left ventricular endocardial potentials always decreased as left ventricular enddiastolic dimension increased. Left ventricular diameters were also different in different animals; the data were, therefore, treated statistically by the z-transformation (Fig. 4). Such analysis shows that a close negative linear relation exists between left ventricular endocardial potentials and left ventricular end-diastolic dimensions when the variances between electrodes and dogs are accounted for.

Average changes in left ventricular endocardial potentials at maximal increase in left ventricular end-diastolic dimension during transfusion, together with changes in haemodynamic levels are summarised in Table 1 . As expected, together with an 


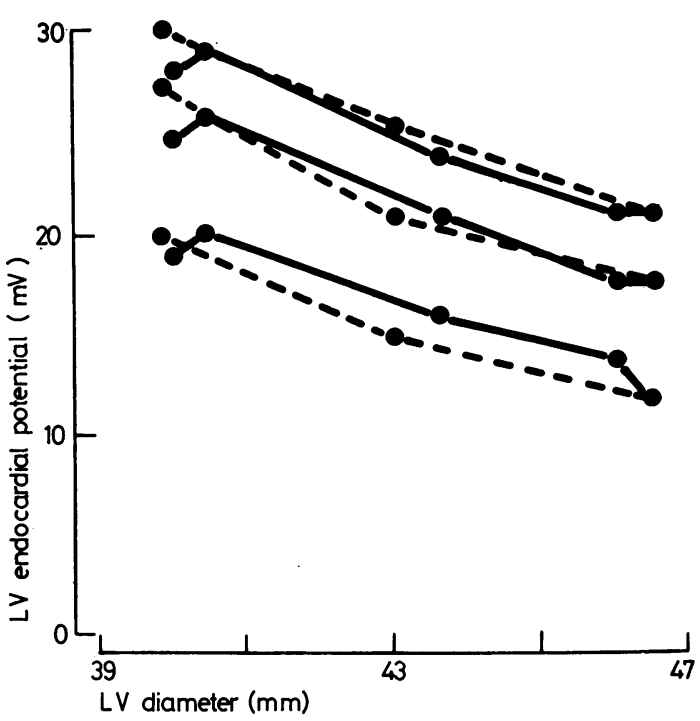

Fig. 2 Changes in left ventricular endocardial potential $(L V E C P)$ recorded from three different electrodes of $L V$ anterior wall during transfusion (solid lines) and withdrawal (broken lines) of blood. Though LVECP recorded from different sites varied in magnitude, LVECP from each site decreased when left ventricular end-diastolic diameter increased and vice versa. Control levels were always regained.

increase in left ventricular end-diastolic dimension, there was a substantial increase in left ventricular systolic and end-diastolic pressures and cardiac output. With an average increase in left ventricular end-diastolic dimension of 11 per cent, left ventricular endocardial potentials decreased by 28 per cent.

\section{LEFT VENTRICULAR VOLUMES AND ENDO- CARDIAL POTENTIALS}

In 7 dogs, changes in left ventricular end-diastolic volume and left ventricular endocardial potentials were determined during transfusion and blood withdrawal. Increase in left ventricular end-diastolic volume was associated with a decrease in left ventricular endocardial potentials, and vice versa. In addition in this series, the absolute left ventricular volumes and endocardial potentials varied considerably in different dogs. However, in each animal, the relation between changes in left ventricular end-diastolic volume and left ventricular endocardial potentials was linear within the range of changes in left ventricular volumes achieved. The relation between individual measurements of left ventricular endocardial potentials and left ventricular end-diastolic volumes, normalised by

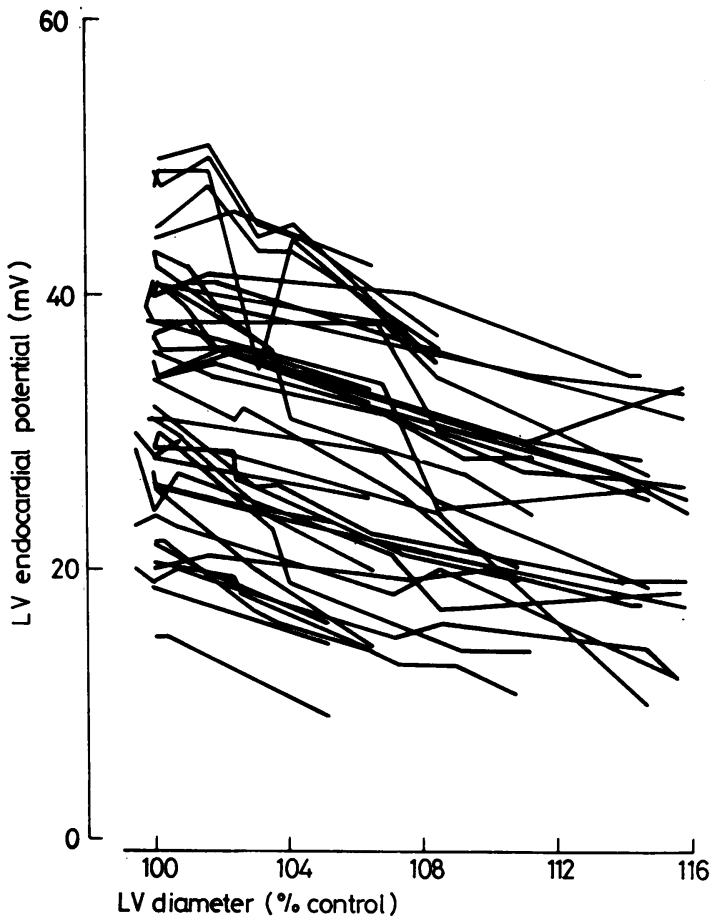

Fig. 3 Relation between changes in left ventricular endocardial potential ( LVECP) and LV end-diastolic diameter during transfusion and withdrawal of blood. Though the absolute magnitude of left ventricular endocardial potential (LVECP) varied from different sites and in different dogs, LVECP always decreased linearly with an increase in diameter.

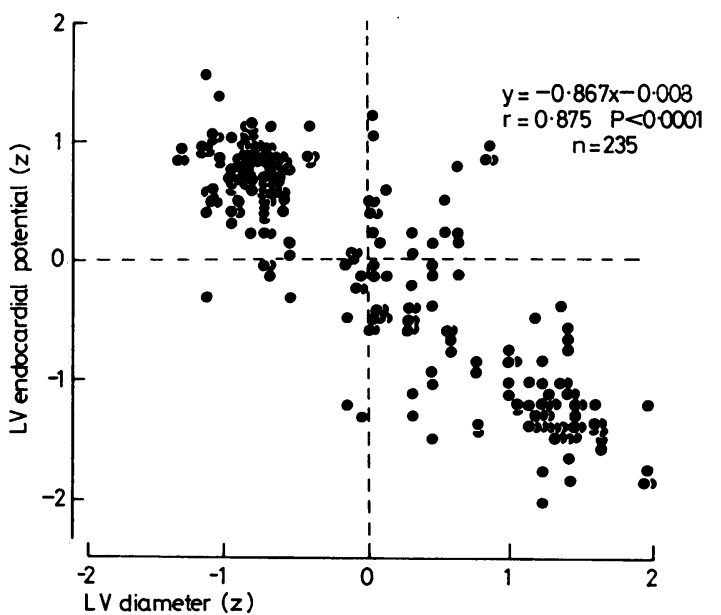

Fig. 4 The data in Fig. 3 as normalised by $z$-transformation. $z=(x-\bar{x}) / S D$ for each electrode and dog. $A$ close linear relation exists between left ventricular endocardial potential (LVECP) and left ventricular end-diastolic dimension (LVEDD). 
Table 1 Effects of blood infusion on endocardial potentials and left ventricular diameter in 11 dogs

\begin{tabular}{lrlll}
\hline & $N$ & Control & Highest load & $P$ \\
\hline LVECP $(\mathrm{mV})$ & 41 & $32.8 \pm 2.5$ & $23.5 \pm 2.3$ & $<0.001$ \\
Area $(\mathrm{mV} \times \mathrm{ms})$ & 35 & $657 \pm 70$ & $498 \pm 68$ & $<0.001$ \\
DUR $(\mathrm{ms})$ & 35 & $44.0 \pm 1.5$ & $43.2 \pm 1.8$ & NS \\
LVEDD $(\mathrm{mm})$ & 11 & $40.09 \pm 1.70$ & $44.56 \pm 1.79$ & $<0.001$ \\
LVEDP $(\mathrm{mmHg})$ & 11 & $4.7 \pm 1.1$ & $16.3 \pm 2.0$ & $<0.001$ \\
LVSP $(\mathrm{mmHg})$ & 11 & $128 \pm 8$ & $152 \pm 7$ & $<0.001$ \\
CO $(\mathrm{ml} / \mathrm{min})$ & 4 & $3660 \pm 120$ & $6280 \pm 1020$ & $<0.05$ \\
\hline
\end{tabular}

$\mathrm{N}$, number of observations; LVECP, left ventricular endocardial potential; Area, area of the total QRS complex; DUR, duration of the QRS complex; LVEDD, left ventricular end-diastolic diameter ; LVEDP, left ventricular end-diastolic pressure; LVSP, left ventricular systolic pressure; $\mathrm{CO}$, cardiac output

z-transformation, is illustrated in Fig. 5. A negative linear relation was observed between left ventricular endocardial potential and left ventricular enddiastolic volume. Average changes in haemodynamic values at maximum left ventricular end-diastolic volume are summarised in Table 2 . With an average increase in left ventricular end-diastolic volume of 152 per cent left ventricular endocardial potentials decreased by 59 per cent. Left ventricular systolic and end-diastolic pressures and cardiac output increased significantly during transfusion.

LEFT VENTRICULAR ANTERIOR AND POSTERIOR ENDOCARDIAL POTENTIALS

In 3 dogs, concurrent changes in left ventricular endocardial potentials recorded from anterior and

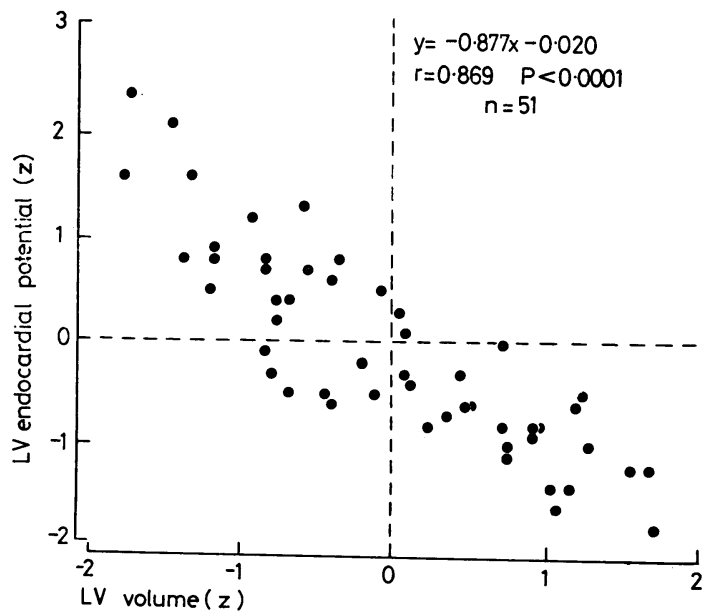

Fig. 5 Relation between left ventricular endocardial potential (LVECP) and left ventricular end-diastolic volume. The data were normalised by $z$-transformation as in Fig. 4. A close linear relation exists between LVECP and left ventricular end-diastolic volume (LVEDV).
Table 2 Effects of blood infusion on endocardial potentials in left and right ventricles in 7 dogs

\begin{tabular}{llll}
\hline & Control & Highest load & $P$ \\
\hline LVECP $(\mathrm{mV})$ & $36.2 \pm 6.6$ & $14.9 \pm 4.3$ & $<0.002$ \\
LVEDV $(\mathrm{ml} / \mathrm{kg} \mathrm{bw})$ & $1.36 \pm 0.25$ & $3.43 \pm 0.58$ & $<0.01$ \\
LVEDP $(\mathrm{mmHg})$ & $3.5 \pm 1.3$ & $22.9 \pm 3.5$ & $<0.001$ \\
LVSP $(\mathrm{mmHg})$ & $112 \pm 7$ & $133 \pm 5$ & $<0.01$ \\
RVECP $(\mathrm{mV})$ & $25.0 \pm 3.6$ & $10.8 \pm 1.7$ & $<0.001$ \\
RVEDP $(\mathrm{mmHg})$ & $4.0 \pm 1.4$ & $11.6 \pm 1.7$ & $<0.001$ \\
HR (beats/min) & $175 \pm 12$ & $149 \pm 9$ & NS \\
CO (ml/min) & $1880 \pm 420$ & $5000 \pm 440$ & $<0.001$ \\
\hline
\end{tabular}

LVECP, left ventricular endocardial potential ; LVEDV, left ventricular end-diastolic volume; RVECP, right ventricular endocardial potential; RVEDP, right ventricular end-diastolic pressure; HR, heart rate; other symbols and abbreviations as in Table 1.

posterolateral walls were determined during increase and decrease of left ventricular volume. In all 3 dogs, left ventricular endocardial potentials, recorded both from anterior and posterolateral wall, behaved similarly during left ventricular volume changes, though the absolute magnitude of

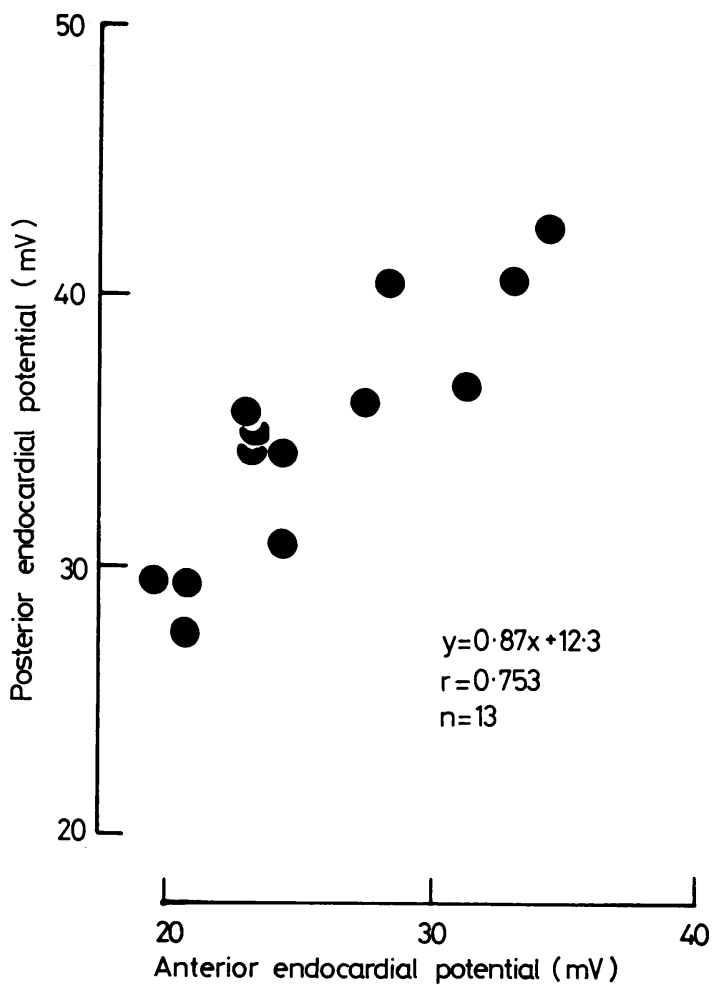

Fig. 6 Relation between changes in left ventricular endocardial potential (LVECP) recorded from left ventricular anterior and posterolateral wall in one representative experiment during transfusion and withdrawal of blood. Both anterior and posterolateral LVECP behaved similarly. 
left ventricular endocardial potentials was different. The relation between left ventricular endocardial potentials recorded from the anterior and posterior walls of the left ventricle in one experiment is shown in Fig. 6. Left ventricular endocardial potentials recorded from both sites changed similarly, indicating that changes in these potentials during changes in left ventricular volume were independent of the site of recording of endocardial potentials.

CHANGES IN AREA, DURATION, AND MAXIMUM AMPLITUDE OF ENDOCARDIAL POTENTIAL

To determine whether there was a gross change in intraventricular conduction, changes in duration and area of the endocardial QRS complex were determined in 35 of the 41 electrodes and compared with the maximum amplitude of left ventricular endocardial potentials. There was no significant change in duration, and the area of the QRS complex decreased proportionally to the decrease in left ventricular endocardial potentials when left ventri- cular end-diastolic dimension increased during transfusion (Table 1). These findings indicate that a reduction in left ventricular endocardial potentials during left ventricular volume expansion probably was not the result of any change in intraventricular conduction.

COMPARISON OF RIGHT AND LEFT VENTRICULAR ENDOCARDIAL POTENTIALS

Right ventricular endocardial potential like left ventricular endocardial potential decreased in amplitude during transfusion and increased during blood withdrawal (Fig. 7). In 7 dogs, changes in right and left ventricular endocardial potentials were compared during ventricular volume changes. Though the absolute magnitudes in different dogs were variable, changes in right ventricular endocardial potential paralleled those in the left ventricle. The relation between individual values of right and left ventricular endocardial potentials normalised by $\mathrm{z}$-transformation is shown in Fig. 8; the correla-
RV endocardial potential

\section{LV}

pressure

RV

pressure

LV

pressure

LV diameter

Aortic pressure
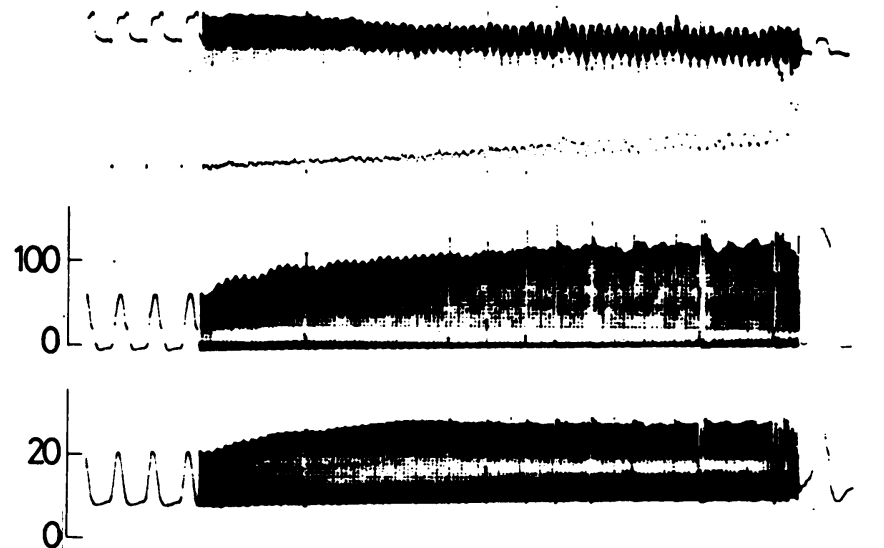

Fig. 7 Decrease in right ventricular $(R V)$ endocardial potential associated with an increase in left ventricular $(L V)$ diameter during transfusion in one experiment. Increase in right ventricular systolic and end-diastolic and peak left ventricular systolic pressures were also observed. N 


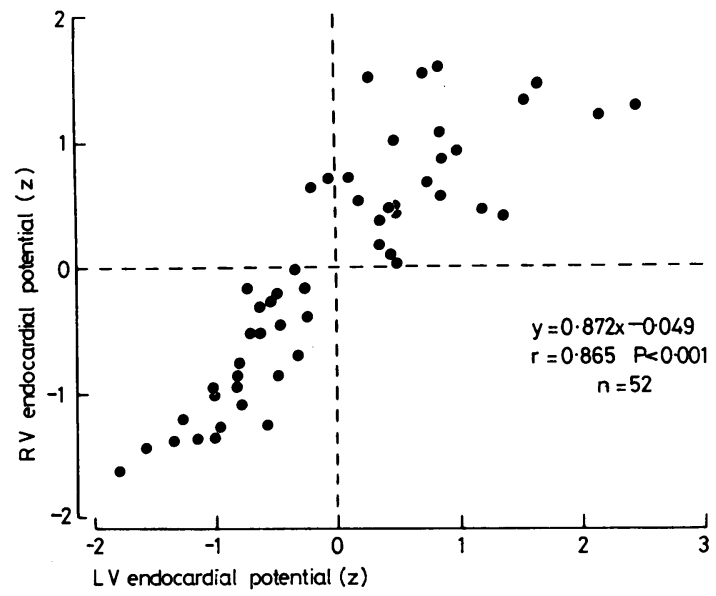

Fig. 8 Relation between changes in right and left ventricular endocardial pressures (RVECP and LVECP) during transfusion and blood withdrawal. Data were normalised statistically by z-transformation. Close relation exists between changes in RVECP and LVECP.

tion indicates that relative changes between them were similar.

In order to assess the effects of an isolated increase in right ventricular volume on right and left ventricular endocardial potentials, temporary constriction of the pulmonary artery was produced in one experiment (Fig. 9). Right ventricular pressure increased significantly indicating an increase in right ventricular end-diastolic volume, while left ventricular end-diastolic dimension did not change significantly. Right ventricular endocardial potentials recorded from three different sites decreased slightly in all three electrodes; left ventricular endocardial potentials, however, remained unchanged. Thus, it appears that changes in one ventricular volume in isolation may be reflected by changes in endocardial potential recorded from the same ventricle. During vena caval obstruction in the same dog (Fig. 10), there was an increase both in right and left ventricular endocardial potentials as the ventricular pressures and left ventricular end-diastolic dimension decreased.

EFFECT OF CHANGES IN HAEMATOCRIT ON ENDOCARDIAL POTENTIALS

In all experiments, the haematocrit remained virtually constant during the blood transfusion and withdrawal procedures (range 45 to $38 \%$ ). The effect of pronounced changes in haematocrit on endocardial potentials was studied in one particular experiment. The haematocrit was gradually reduced without changing left ventricular end-diastolic dimension by saline exchange infusion (Fig. 11).

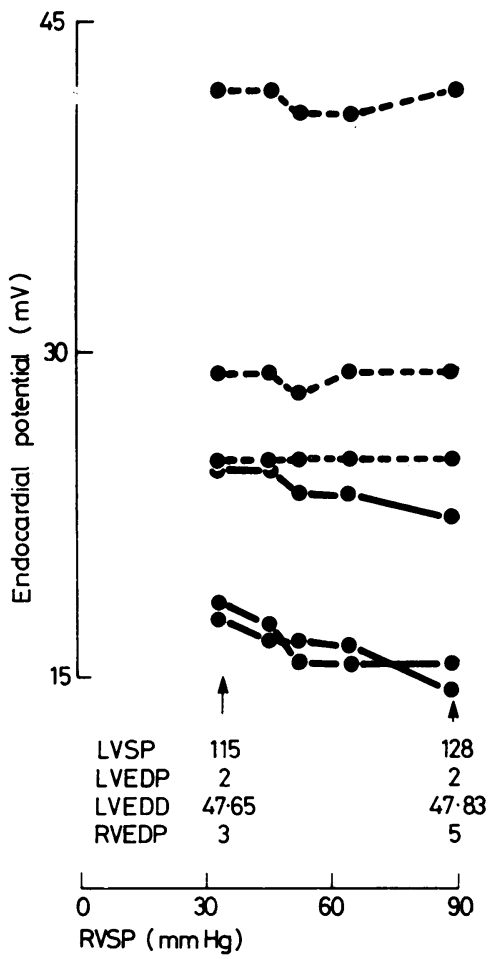

Fig. 9 Effect of pulmonary artery constriction on left ventricular endocardial potentials (broken lines) and right ventricular endocardial potentials (solid lines). Right ventricular endocardial potentials (RVECP) decreased slightly, associated with an increase in right ventricular end-diastolic pressure (RVEDP). There was no significant change either in LVECP or LVEDD. LVSP = left ventricular systolic pressure; LVEDP = left ventricular end-diastolic pressure; LVEDD = left ventricular enddiastolic diameter; $R V E D P=$ right ventricular enddiastolic pressure; $R V S P=$ right ventricular systolic pressure.

There was little or no change in endocardial potentials when haematocrit decreased from 43 to 35 per cent; however, with a further decrease in haematocrit, both right and left ventricular endocardial potentials decreased significantly.

\section{Discussion}

The present study shows that acute changes in ventricular volumes are associated with changes in ventricular endocardial potentials. In all dogs in this study left ventricular endocardial potentials decreased as the ventricular volume increased, and increased as ventricular volume was decreased.

The absolute magnitude of left ventricular endocardial potentials in different dogs varied considerably. In addition, the magnitude of left 


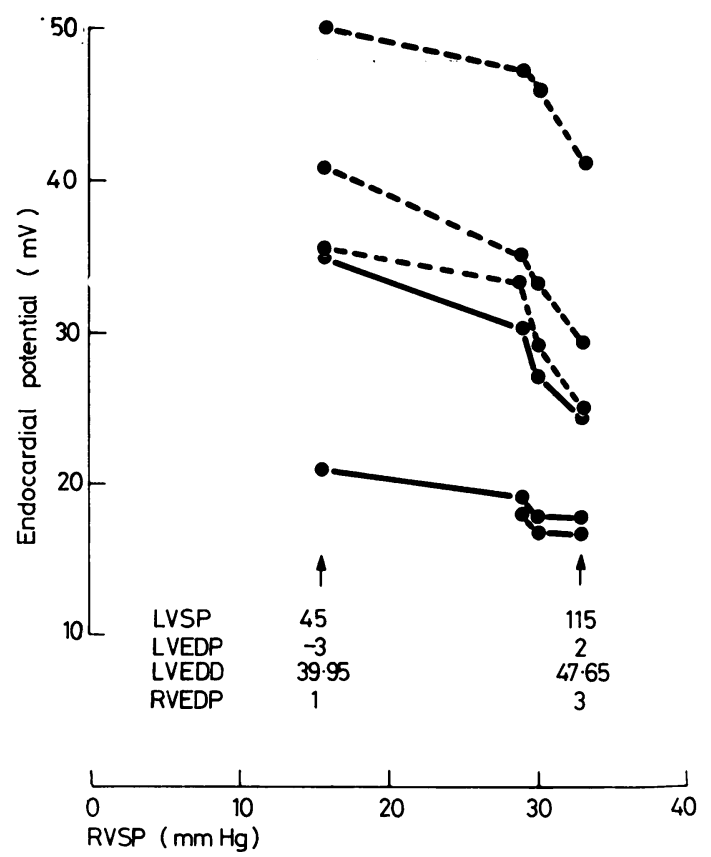

Fig. 10 Effect of vena cava obstruction on $L V$ endocardial potentials (broken lines) and $R V$ endocardial potentials (solid lines). Other symbols as in Fig. 9. Reduced LVEDP, $L V E D D$, and RVEDP were associated with an increase in both $L V E C P$ and $R V E C P$.

ventricular endocardial potentials recorded from different sites in the same ventricle varied. However, irrespective of the initial magnitude of endocardial potentials and irrespective of their site of recording, they always decreased as the ventricular volume increased and vice versa. Control left ventricular end-diastolic volume was also variable in different dogs. However, when these variables were normalised by $z$-transformation, an inverse linear relation was observed between changes in left ventricular end-diastolic volume and left ventricular endocardial potential. These findings suggest that for a given change in left ventricular end-diastolic volume, a proportional change in left ventricular endocardial potential may be expected, irrespective of the initial value of either.

Because of the problems of accurately determining left ventricular volumes by the thermodilution technique, changes in left ventricular diameter were also measured from ultrasonic crystals implanted in the ventricular walls. Changes in enddiastolic diameter during transfusion or blood withdrawal were then correlated with changes in endocardial potential. This technique allowed for evaluation of beat-to-beat changes in left ventricular end-diastolic dimension and endocardial potential.

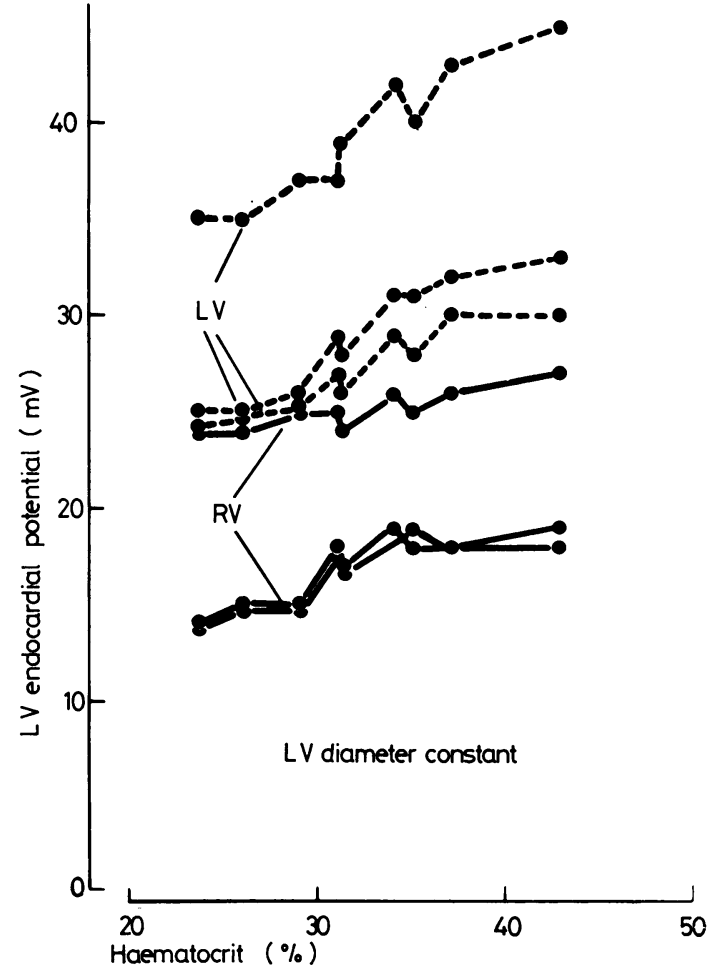

Fig. 11 Effect of reduced haematocrit on ventricular endocardial potentials. Endocardial potentials were recorded from three different sites from both left ventricle (broken line) and right ventricle (solid line). LV enddiastolic diameter was kept constant. With modest decrease in haematocrit there was little or no decrease in endocardial potential (ECP); with a pronounced decrease in haematocrit, however, there was a significant decrease in ECP.

Results showed a negative linear relation between left ventricular end-diastolic dimension and left ventricular endocardial potential: thus, when there was an increase in the former, latter fell and vice versa. The relation was maintained irrespective of the site of recording of left ventricular endocardial potential. In this study, when changes in left ventricular volume were determined by thermodilution, an average decrease in left ventricular endocardial potential of 59 per cent was associated with an increase in left ventricular end-diastolic volume of 152 per cent. When changes in left ventricular diameter were determined by ultrasound technique, an average decrease in left ventricular endocardial potential of 28 per cent was associated with an increase in left ventricular end-diastolic dimension of 11 per cent. Thus, there was a larger increase in left ventricular end-diastolic volume than would be predicted from the data 
relating endocardial potential and end-diastolic dimension on the assumption that the volume of the ventricle is proportional to the cube of the diameter. This discrepancy is not readily explicable, but mixing of indicator within the ventricle probably becomes less adequate at greater left ventricular end-diastolic volume and possibly produced an overestimation of this (Hallerman et al., 1963; Carleton et al., 1966). It is also possible that the changes in the anterior-posterior diameter do not reflect changes in ventricular size and movement. However, despite difficulties with assessing left ventricular volume precisely, the present study shows a clear relation between left ventricular endocardial potential and left ventricular dimensions.

This study also shows that endocardial potential recorded from the right ventricle closely follows changes in left ventricular endocardial potential. During transfusion and blood withdrawal, an increase or decrease in left ventricular endocardial potential was associated with similar changes in right ventricular endocardial potential. Though the absolute magnitude of right and left ventricular endocardial potential was variable, there was a good relation between changes in them. However, with pulmonary constriction associated with raised right ventricular pressure, and presumably increased right ventricular end-diastolic volume, only right ventricular endocardial potential decreased, though slightly. There was no change in either left ventricular end-diastolic dimension or left ventricular endocardial potential. It is, therefore, likely that an increase in one ventricular volume in isolation would predominantly influence the endocardial potential recorded from this ventricle.

Although the present study shows that changes in ventricular volumes and dimension influence the amplitude of the endocardial QRS complex, the precise mechanism of these changes remains unclear. It has been demonstrated that changes in ventricular volume influence the amplitude of surface $Q R S$ potentials, particularly their initial ventricular depolarisation force generated by radial dipoles (Voukydis, 1974). In the present study, however, the changes in endocardial QRS amplitude during changes in ventricular volume were opposite to the reported changes in surface potentials. It appears, therefore, that the mechanisms of changes in surface and endocardial potentials may be dissimilar. In the present study with open-chest dogs, surface QRS potentials could not be recorded so that the relation between surface and endocardial potentials could not be explored. In some dogs, however, epicardial potentials over the anterior left ventricular surface were recorded concurrently with left ventricular anterior endocardial potentials.
LV epicardial potential

LV
pressure

RV pressure

LV pressure

LV
diameter

diameter
diam 100

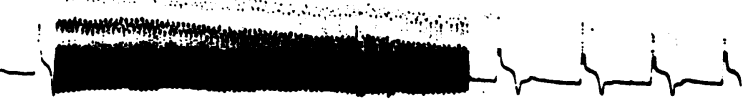

pressure

Aortic pressure

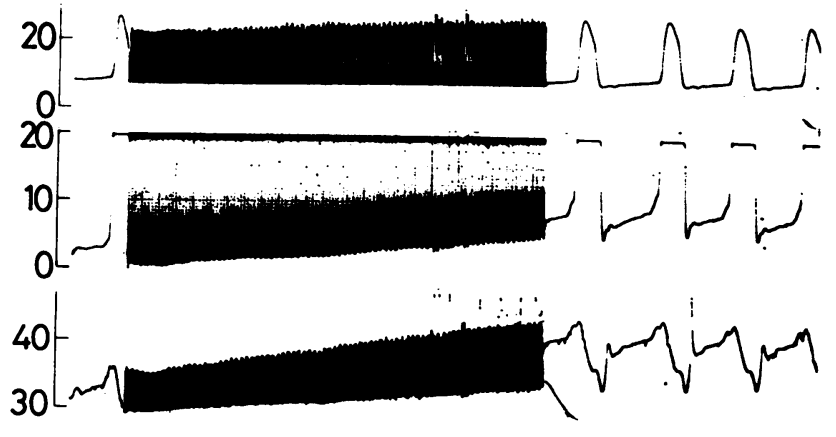

Fig. 12 Decrease in epicardial potentials associated with increase in left ventricular $(L V)$ diameter during blood transfusion 
Similar to changes in endocardial potentials, epicardial potentials also decreased during volume expansion (Fig. 12).

A possible explanation is suggested by geometrical considerations. We assume that an endocardial electrode is primarily influenced by the adjacent myocardium. As left ventricular end-diastolic volume increases, wall thickness decreases, thus reducing the mass of myocardium represented by the exploring electrode. If a cube of tissue with a constant volume lengthens in its two surface dimensions by 11 per cent, as was measured in this study (an $11 \%$ increase in diameter corresponds to an $11 \%$ increase in segment length), it must thin by 19 per cent, the same order of magnitude as the observed 28 per cent reduction in left ventricular endocardial potential. Preferential current shunting through blood might reduce endocardial potential as ventricular volume increases. These considerations suggest the importance of geometric factors, though further investigations are needed to elucidate the precise mechanism for the relation between endocardial potential and ventricular dimensions.

One practical result of these observations is that an increase in epicardial or endocardial QRS amplitudes should not necessarily be regarded as indicative of improvement or worsening of myocardial ischaemia as has been recently proposed (Hillis et al., 1976). Such changes may also be partly the result of changes in ventricular volume not associated with changes in myocardial injury. Reduction of endocardial potential in acute myocardial infarction may also be caused by an increase in end-diastolic volume which frequently accompanies recent infarction. It is also possible, however, that infarction per se decreases endocardial potential. Without further studies, the relative influence of myocardial infarction and associated changes in ventricular volume cannot be determined.

A reduction in haematocrit can augment the QRS amplitude of surface electrograms (Rosenthal et al., 1971; Hodkin et al., 1977). These changes in surface potentials are probably related to changes in conductivity of the blood within the ventricular cavity. This can distort the cardiac vector recorded from the body surface since it tends to produce augmentation of voltages produced by radially oriented forces and suppression of tangential components ('Brody effect') (Brody, 1956). Possible influence of changes in haematocrit explaining the reduction in endocardial potential during volume expansion of the heart can, however, be excluded in this study because only extremely large reductions in haematocrit were associated with significantly reduced endocardial potentials in our setting.

It needs to be emphasised that this study has only shown the influence of acute changes in ventricular volume on endocardial potentials. The effect of chronic changes in ventricular volume on endocardial potentials remains unknown. The fact that acute changes in ventricular volume conspicuously influence the magnitude of endocardial potentials may at least partly explain the clinical observation that endocardial potentials are lower in patients with acute heart failure. With improvement in heart failure and a decrease in end-diastolic volume, the magnitude of the endocardial potential should also increase. Preliminary clinical data support the relation between acute changes in ventricular volume and ventricular endocardial potentials. During a Valsalva manoeuvre or after the administration of sublingual glyceryl trinitrate, interventions that decrease left ventricular enddiastolic volume, right ventricular endocardial potentials increased in magnitude (Chatterjee et al., 1975). Immediately after left ventricular contrast angiography, right ventricular endocardial potentials decreased as might be expected since left ventricular end-diastolic volumes probably increased (Chatterjee et al., 1975). Further studies are required, however, to establish the precise relation between changes in ventricular volume and endocardial potential in man.

In conclusion, this study shows that acute changes in ventricular volume considerably influence the magnitude of ventricular endocardial potentials. There is a negative linear relation between left ventricular end-diastolic volume and ventricular endocardial potentials, though the precise mechanism remains unclear. Since changes in right ventricular endocardial potentials also reflect changes in left ventricular end-diastolic volume (except in circumstances where there are discordant changes in right and left ventricular volumes), monitoring changes in right ventricular endocardial potentials may have a potential clinical role in detecting acute changes in left ventricular enddiastolic volume.

The authors thank $\mathrm{Dr}$ Arturo Anonas and $\mathrm{Mr}$ Jerome Howard for technical assistance; and Dr Stanton A. Glantz for his help with statistical analysis.

\section{References}

Brody, D. A. (1956). A theoretical analysis of intracavitary blood mass influence on the heart-lead relationship. Circulation Research, 4, 731-738.

Carleton, R. A., Bowyer, A. F., and Graettinger, J. S. (1966). Overestimation of left ventricular volume by the indicator dilution technique. Circulation Research, 18, 248-256.

Chatterjee, K., Harris, A., Davies, G., and Leatham, A. (1970). Fall of endocardial potentials after acute myocardial

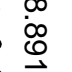
음

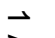


infarction. Lancet, 1, 1308-1312.

Chatterjee, K., and Rouse, W. (1971). Ventricular endocardial potentials after experimental coronary artery occlusion in dogs. American Heart fournal, 82, 352-361.

Chatterjee, K., Sutton, R., and Davies, J. G. (1968). Low intracardiac potentials in myocardial infarction as a cause of failure of inhibition of demand pacemakers. Lancet, 1, 511.

Chatterjee, K., Sutton, G. C., and Miller, G. A. H. (1972). Right ventricular endocardial potential in acute massive pulmonary embolism. British Heart fournal, 34, 271-273.

Chatterjee, K., Tyberg, J. V., Stowe, D., Ratshin, R., Moores, W., Ostlund, J., and Parmley, W. W. (1975). The use of ventricular endocardial $Q R S$ amplitude as a new technique for monitoring changes in left ventricular volumes (abstract). Circulation, 51 and 52, Suppl. II, 168.

Hallerman, F. J., Rastelli, G. C., and Swan, H. J. C. (1963). Comparison of left ventricular volumes by dye dilution and angiographic methods in the dog. American fournal of Physiology, 204, 446-450.

Hillis, L. D., Askenazi, J., Braunwald, E., Radvany, P., Muller, J. E., Fishbein, M. C., and Maroko, P. R. (1976). Use of changes in the epicardial QRS complex to assess interventions which modify the extent of myocardial necrosis following coronary artery occlusion. Circulation, 54, 591-598.

Hodkin, B. C., Millard, R. W., and Nelson, C. V. (1977). Effect of hematocrit on electrocardiographic potentials and dipole moment of the pig. American fournal of Physiology, 232, H406-H410.
Holt, J. P. (1956). Estimation of the residual volume of the ventricle of the dog's heart by two indicator dilution techniques. Circulation Research, 4, 187-195.

Nie, N. H., Hull, C. H., Jenkins, J. G., Steinbrenner, K., and Bent, D. H. (1975). Statistical Package for the Social Sciences, 2nd ed. McGraw-Hill, New York.

Parker, B., Furman, S., and Escher, D. J. W. (1969). Input signals to pacemakers in a hospital environment. Annals of the New York Academy of Sciences, 167, 823-834.

Rosenthal, A., Restieaux, N. J., and Feig, S. A. (1971). Influence of acute variations in hematocrit on the QRS complex of the Frank electrocardiogram. Circulation, 44, 456-465.

Theroux, P., Franklin, D., Ross, J., Jr., and Kemper, W. S. (1974). Regional myocardial function during coronary artery occlusion and its modification by pharmacologic agents in the dog. Circulation Research, 35, 896-908.

Voukydis, P. C. (1974). Effect of intracardiac blood on the electrocardiogram. New England fournal of Medicine, 291, 612-616.

Zar, J. H. (1974). Biostatistical Analysis, p. 239. Prentice-Hall, Englewood Cliffs, N.J.

Requests for reprints to Professor Kanu Chatterjee, Cardiovascular Division, Coronary Care Unit, Room 1186 Moffitt Hospital, University of California, San Francisco, California 94143, U.S.A. 